\title{
Temperature Dependence Study of Mesa-Type InGaAs/InAlAs Avalanche Photodiode Characteristics
}

\author{
Jack Jia-Sheng Huang, ${ }^{1,2}$ H. S. Chang, ${ }^{2}$ Yu-Heng Jan, ${ }^{1,2}$ C. J. Ni, ${ }^{2}$ \\ H. S. Chen, ${ }^{2}$ and Emin Chou $^{2}$ \\ ${ }^{1}$ Source Photonics, 8521 Fallbrook Avenue, Suite 200, West Hills, CA 91304, USA \\ ${ }^{2}$ Source Photonics, No. 46, Park Avenue 2nd Rd., Science-Based Industrial Park, Hsinchu, Taiwan \\ Correspondence should be addressed to Jack Jia-Sheng Huang; jack.huang@sourcephotonics.com
}

Received 19 September 2016; Revised 5 December 2016; Accepted 22 December 2016; Published 12 January 2017

Academic Editor: Jung Y. Huang

Copyright (c) 2017 Jack Jia-Sheng Huang et al. This is an open access article distributed under the Creative Commons Attribution License, which permits unrestricted use, distribution, and reproduction in any medium, provided the original work is properly cited.

\begin{abstract}
Avalanche photodiodes (APDs) are key optical receivers due to their performance advantages of high speed, high sensitivity, and low noise. The most critical device parameters of APD include the avalanche breakdown voltage and dark current. In this work, we study the temperature dependence of the breakdown voltage and dark current of the mesa-type APD over a wide temperature range of $20-145^{\circ} \mathrm{C}$. We institute an empirical model based on impact ionization processes to account for the experimental data. It is shown that highly stable breakdown characteristics of mesa-type APD can be attained with the optimization of the multiplication layer design. We have achieved excellent stability of avalanche breakdown voltage with a temperature coefficient of $0.017 \mathrm{~V} /{ }^{\circ} \mathrm{C}$. The temperature dependence of dark current is attributed to generation-recombination mechanism. The bandgap energy is estimated to be about $0.71 \mathrm{eV}$ based on the temperature variation of dark current, in good agreement with the value for InGaAs.
\end{abstract}

\section{Introduction}

Semiconductor photodiodes are important components for the high-sensitivity, low-noise receivers and detectors deployed in the 2.5, 10, and 25G optical communication systems such as passive optical network (PON) and local area networks (LAN) [1]. Among the photodiode portfolio, APDs are attractive devices due to the significant improvement in photoreceiver sensitivity compared with traditional p$\mathrm{i}$-n (PIN) photodiodes [2]. By adding the multiplication layer, the avalanche photodiodes combine the detection and amplification properties simultaneously.

Recently, 10G/25G APD has drawn increasing interest in the commercial and military applications due to the high-bandwidth and low-noise performance advantages. In order to achieve the high bandwidth, a mesa structure with coplanar $\mathrm{P}$ and $\mathrm{N}$ electrodes is typically employed [3-6]. For APD, there are two critical device parameters for the reverse bias operation. The first parameter is the avalanche breakdown voltage associated with the multiplication layer.
The breakdown voltage is typically measured at reverse current of $10 \mu \mathrm{A}$. The avalanche breakdown has been theoretically formulated to study the impact ionization coefficients of electrons and holes [7-9]. The second parameter is the dark current that is typically measured at reverse bias below the breakdown voltage. Since these two parameters strongly influence the device performance of the APD, it is important to understand the temperature dependence of these two parameters. A good knowledge of the temperature dependence is critical for the design of robust APD that can maintain stable performance when subject to temperature fluctuations.

In this paper, we study the temperature dependence of breakdown voltage and dark current of the mesa-type APD. We experimentally measure the temperature variations of the breakdown voltage and dark current and compare the data with the modelling results based on depletion and impact ionization processes. We discuss the estimation of the energy bandgap using the dark current from the generation current model. 


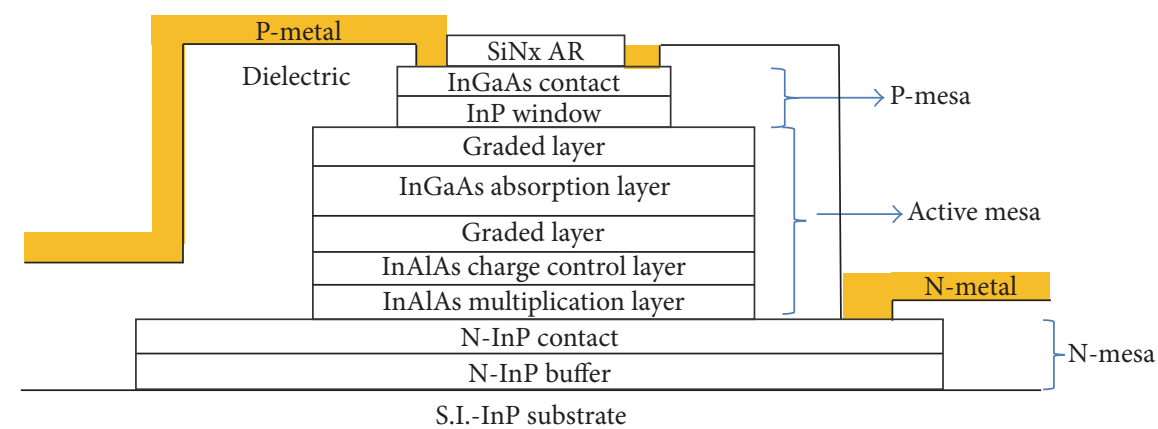

FIGURE 1: Schematic of the mesa-type APD structure used in the temperature dependence study. In the design, the InGaAs absorption layer is on top of the InAlAs charge control and multiplication layers.

\section{Experimental}

Figure 1 shows the schematic of the mesa-type APD structure. The APD devices used in the modelling and experimental studies included the $\mathrm{N}$-mesa at the bottom, the active region in the middle, and the P-mesa at the top [10]. The $\mathrm{N}$ mesa consisting of the N-InP buffer and contact layers was grown on top of semi-insulating (SI) InP substrate. The active region consisted of the InAlAs multiplication, the InAlAs charge control, the graded InGaAs/InAlAs, and the InGaAs absorption layers. The InAlAs multiplication layer was undoped with a thickness of about $160 \mathrm{~nm}$. The InAlAs charge control layer was p-type doped $\left(\sim 1.0-1.1 \times 10^{18} \mathrm{~cm}^{-3}\right)$. The InGaAs absorption layer was undoped with a thickness of about $1200 \mathrm{~nm}$. The P-mesa consisted of the $\mathrm{p}$-InP window and $\mathrm{p}$-InGaAs contact layers. To achieve the $10 \mathrm{G}$ or $25 \mathrm{G}$ high speed, the mesa-type APD was formed where both $\mathrm{P}$ and $\mathrm{N}$ electrodes were on the top surface. The p-contact was made by forming the metal ring immediately outside the antireflective (AR) window. The p-ring was connected to the outside $\mathrm{p}$-pad by the metal bridge. The $\mathrm{n}$-metal contact was connected to the N-mesa. For the passivation, the low-k dielectric material such as polyimide was used to reduce the capacitance.

The DC characteristics including the reverse current versus voltage (IV) test was measured. The IV as a function of temperature was also tested to study the temperature dependence of breakdown voltage and dark current.

\section{Results and Discussions}

The typical reverse IV of the mesa-type APD is shown in Figure 2. On the IV curve, there are two transitions along the reverse voltage at about $10 \mathrm{~V}$ and $32 \mathrm{~V}$, respectively. When the reverse voltage is applied to the device, the InAlAs multiplication layer begins to be depleted first. The first transition at $10 \mathrm{~V}$ corresponds to the punch-through voltage at which electric field depletes the InAlAs multiplication and $i$-InGaAs absorption layers [11]. The punch-through voltage related to the absorption layer is denoted as $V_{\mathrm{pt}}$. The second transition represents the avalanche breakdown voltage that is also a critical device parameter for APD. The breakdown is typically determined by the InAlAs multiplication layer. By properly controlling the charge density of the charge control

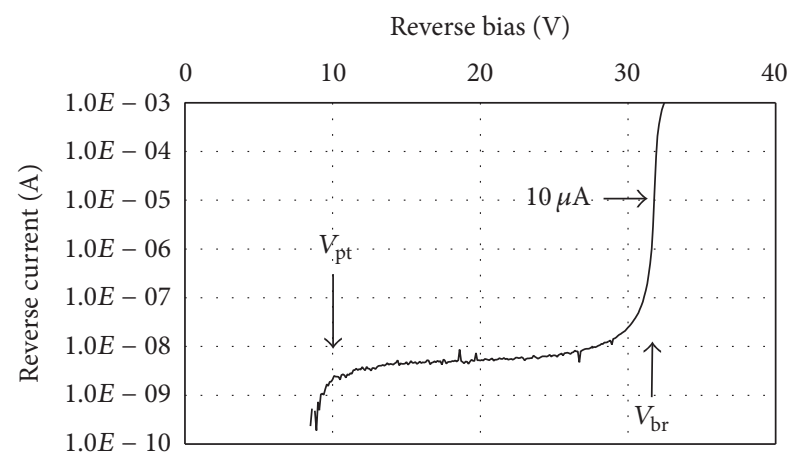

FIGURE 2: A typical IV characteristics of InGaAs/InAlAs mesa-type APD. The IV is measured in reverse polarity at $20^{\circ} \mathrm{C}$.

layer, sufficient electric field can be reached to achieve a good avalanche gain while keeping the tunneling and impact ionization away from the InGaAs absorption layer. In this case, the breakdown voltage taken at $10 \mu \mathrm{A}$ is estimated to be $31.7 \mathrm{~V}$. Between the two transitions, the reverse current is of technological importance for optical detection. The dark current is usually referred to the reverse current at $0.9 \mathrm{~V}_{\mathrm{br}}$. In this case, the dark current is estimated to be about $12.1 \mathrm{nA}$ at $20^{\circ} \mathrm{C}$.

In the following, we study the breakdown voltage and dark current as a function of temperature extensively. We discuss the temperature dependence and compare the experimental data with the modelling results.

3.1. Avalanche Breakdown Voltage $V_{\mathrm{br}}$. There are two major types of junction breakdown in semiconductor diodes. The first type is associated with the tunneling breakdown where the tunneling mechanism is dominant for the devices with lower breakdown voltage. The tunneling breakdown process exhibits a negative temperature coefficient [12, 13]. The second is the avalanche breakdown that is the dominant mechanism for large breakdown voltage, as illustrated in Figure 3. At very high electric field $\left(\sim 10^{5}\right.$ to $\left.10^{6} \mathrm{~V} / \mathrm{cm}\right)$, some electrons within the diffusion distance near the depletion layer gain enough energy to create the secondary electronhole pair by raising the electron from the valence band into the conduction band. This excitation process creates an 

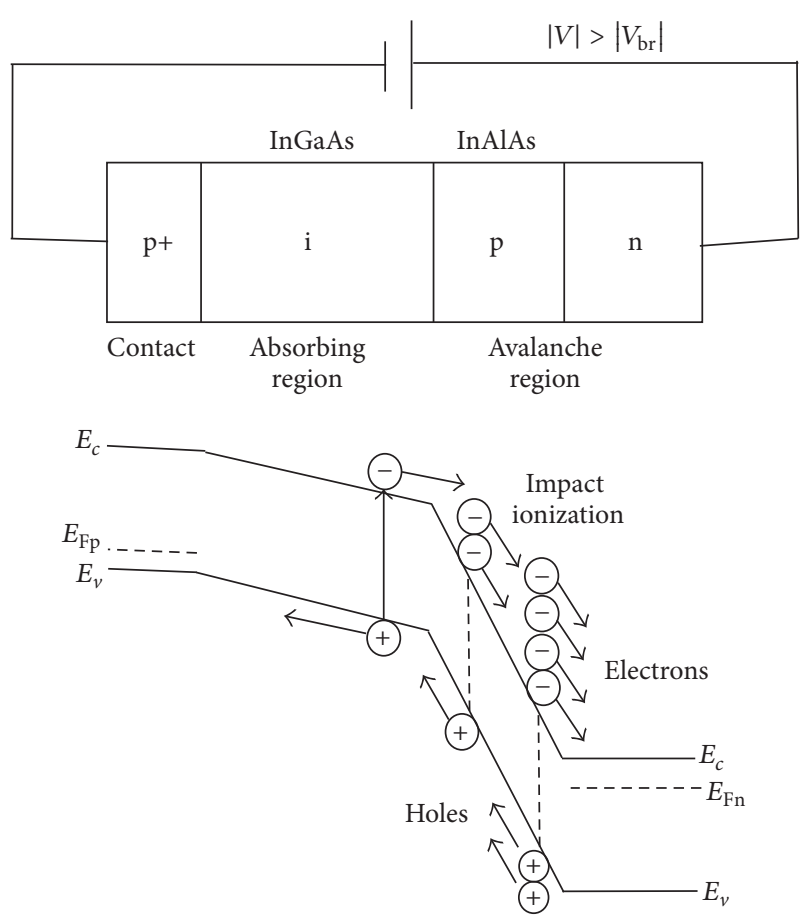

FIGURE 3: Schematics of avalanche breakdown mechanism of APD with simplified energy band diagram at the reverse voltage of $|V|>$ $\left|V_{\mathrm{br}}\right|$. The electron with high enough kinetics energy drifts to the avalanche region and gets accelerated to create a secondary electronhole pair. The secondary electron-hole pair in turn generates another electron-hole pair, leading to snowball avalanche process.

electron-hole pair due to impact ionization. The electrons and holes created by the impact ionization are accelerated by the high electric field. Consequently, the secondary electronhole pair can create even more carriers, leading to a snowball avalanche effect. The avalanche breakdown process typically shows a positive temperature coefficient.

Figure 4 shows the reverse IV as a function of temperature for the mesa-type APD. For APD, the breakdown is due to the avalanche mechanism since the device is operating at high electric field. The increase in ambient temperature is expected to cause the increase in breakdown voltage due to the effect of phonon [14]. As the temperature increases, the population of phonons increases. Thus, a higher electric field and applied voltage are required to reach breakdown in order to overcome the increased carrier cooling caused by phonon scattering [15].

The positive temperature coefficient of the APD has been experimentally found and theoretically described by Tyagi where the breakdown voltage of the Si APD followed a linear expression with temperature [16]. In the modelling of this study, it was assumed that the avalanche breakdown voltage increased linearly with increasing temperature as follows:

$$
V_{\mathrm{br}}(T)=V_{\mathrm{br}}\left(T_{0}\right)\left[1+\alpha\left(T-T_{0}\right)\right],
$$

where $V_{\mathrm{br}}(T)$ is the avalanche breakdown voltage at temperature $T, V_{\mathrm{br}}\left(T_{0}\right)$ is the avalanche breakdown voltage at reference temperature $T_{0}$, and $\alpha$ is the normalized temperature

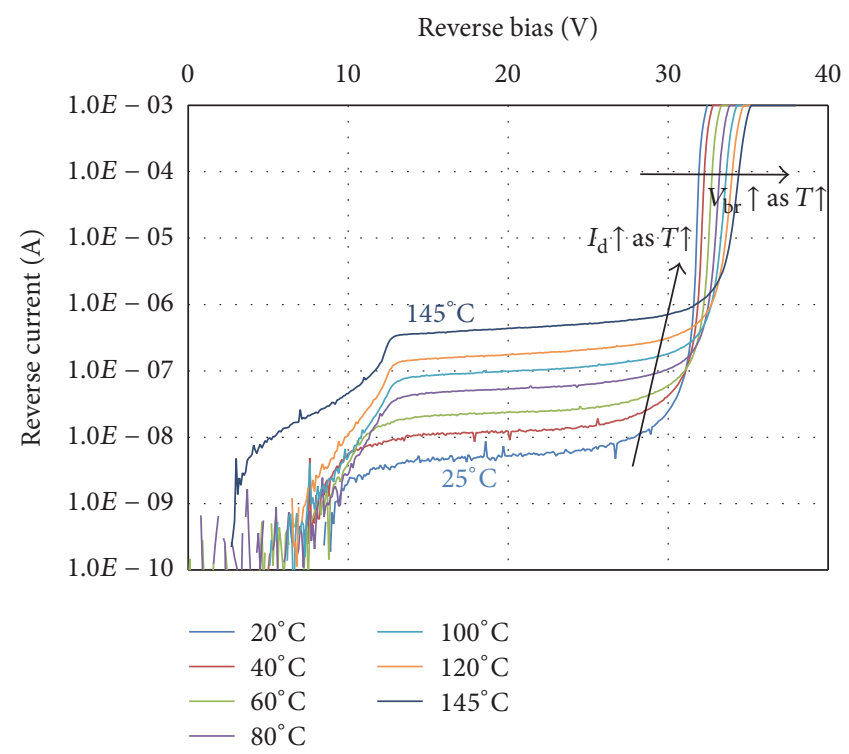

FIgure 4: The reverse IV of mesa-type APD as a function of temperature over a wide range of $20^{\circ} \mathrm{C}$ to $145^{\circ} \mathrm{C}$.

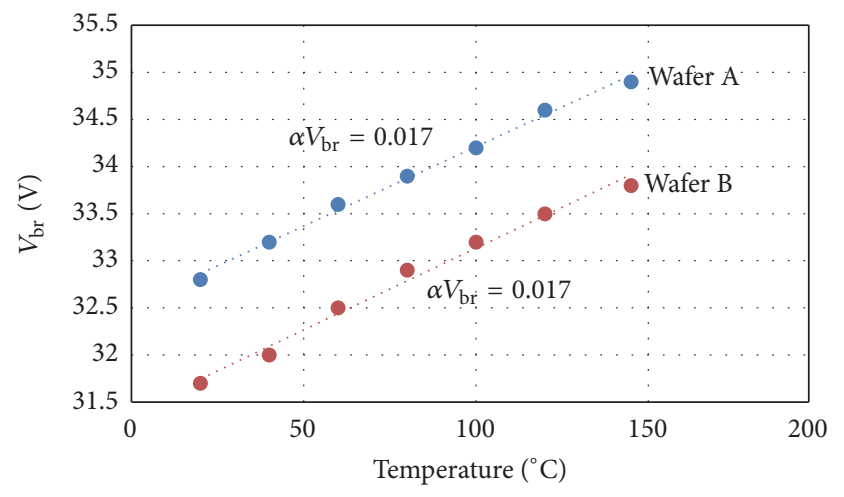

FIGURE 5: The breakdown voltage of mesa-type APD as a function of temperature from $20^{\circ} \mathrm{C}$ to $145^{\circ} \mathrm{C}$. The symbols are experimental data, and the dashed lines are fitting from (2).

coefficient. For the sake of comparison, (1) can be rewritten as follows where the second term shows the temperature coefficient of the breakdown voltage:

$$
V_{\mathrm{br}}(T)=V_{\mathrm{br}}\left(T_{0}\right)+\alpha V_{\mathrm{br}}\left(T_{0}\right)\left(T-T_{0}\right) .
$$

Figure 5 shows the avalanche breakdown voltage as a function of temperature based on two APD wafers with similar structures but from different processing runs. There were several interesting features worth mentioning. First, the two wafers showed similar breakdown voltage. The intercepts at the reference temperature $\left(T_{0}=20^{\circ} \mathrm{C}\right)$ for wafers $\mathrm{A}$ and $\mathrm{B}$ were 32.8 and $31.7 \mathrm{~V}$, respectively. The similarity between the wafers from two different processing runs suggested that the breakdown voltage was largely determined by the epitaxial structure. Secondly, both wafers showed the same temperature coefficient of breakdown voltage where $\alpha V_{\mathrm{br}}$ taken from the slope in Figure 5 was equal to $0.017 \mathrm{~V} /{ }^{\circ} \mathrm{C}$. 
TABLE 1: The temperature dependence of the mesa-type APD measured from $20^{\circ} \mathrm{C}$ to $145^{\circ} \mathrm{C}$.

\begin{tabular}{lccc}
\hline & $V_{\mathrm{br}}\left(T_{0}\right)$ & $\alpha V_{\mathrm{br}}\left(T_{0}\right)$ & $\alpha$ \\
\hline Wafer A & $32.8 \mathrm{~V}$ & $0.017 \mathrm{~V} /{ }^{\circ} \mathrm{C}$ & $5.1 \times 10^{-4} /{ }^{\circ} \mathrm{C}$ \\
Wafer B & $31.7 \mathrm{~V}$ & $0.017 \mathrm{~V} /{ }^{\circ} \mathrm{C}$ & $5.5 \times 10^{-4} /{ }^{\circ} \mathrm{C}$ \\
\hline
\end{tabular}
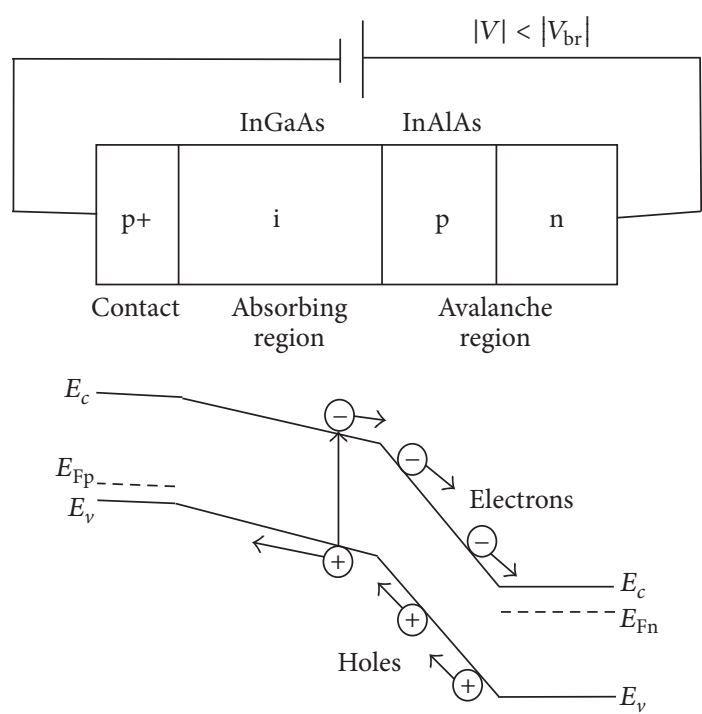

FIGURE 6: Schematics of reverse depletion mechanism of APD with simplified energy band diagram at the reverse voltage of $|V|<\left|V_{\mathrm{br}}\right|$. The avalanche process is not operative below $V_{\mathrm{br}}$. At $10<|V|<$ $\left|V_{\mathrm{br}}\right|$, both i-InGaAs absorption and InAlAs multiplication layers are depleted.

Third, the normalized temperature coefficient $\alpha$ was determined to be $5.1 \times 10^{-4}$ and $5.5 \times 10^{-4 \circ} \mathrm{C}^{-1}$, as shown in Table 1 . The measured value of the normalized temperature coefficient in the mesa-type APD was excellent, lower compared to the reported value of $7.2 \times 10^{-4}{ }^{\circ} \mathrm{C}^{-1}$ [17]. The weak temperature dependence of our mesa-type InGaAs/InAlAs APD brought performance advantage to maintain the gain when the device was subject to temperature fluctuations. We attributed the superior temperature dependence of the avalanche breakdown voltage to the optimized design of the InAlAs multiplication layer thickness and InAlAs charge control layer doping.

3.2. Dark Current $I_{\mathrm{d}}$. Another important device parameter of APD is the dark current [18, 19]. As shown in Figure 4, the dark current typically referred to the reverse current below the breakdown voltage. For different temperature ranging from $20^{\circ} \mathrm{C}$ to $145^{\circ} \mathrm{C}$, the dark current is taken from the reverse voltage of $0.9 V_{\mathrm{br}}$ where $V_{\mathrm{br}}$ increases with the temperature. Figure 6 illustrates the transport of electrons and holes in the energy diagram at the reverse voltage below the avalanche breakdown $\left(|V|<\left|V_{\mathrm{br}}\right|\right)$ and above the punchthrough voltage $(|V|>10)$. In this regime, both $i$-InGaAs absorption and InAlAs multiplication layers are depleted, but the avalanche breakdown in the multiplication layer is not activated.

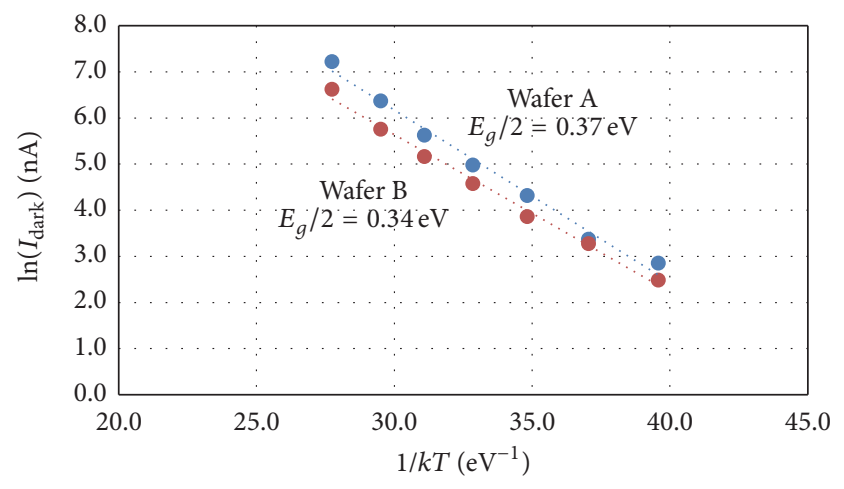

FIGURE 7: The natural logarithm of dark current versus reciprocal of temperature for mesa-type APD. The dark current was measured from $20^{\circ} \mathrm{C}$ to $145^{\circ} \mathrm{C}$.

It has been found that the dark current increases with increasing temperature due to the influence of bandgap energy [20]. We note that the generation-recombination component of the dark current has a temperature dependence. Therefore, the bandgap could be estimated based on the temperature dependence of the dark current. The generation current can be expressed in (3) where $A_{\mathrm{pn}}$ is the area of the $\mathrm{p}-\mathrm{n}$ junction, $W$ is the width of the depletion region, $q$ is the carrier charge, $\sigma$ is the carrier capture cross section, $v_{\text {th }}$ is the carrier thermal velocity, $N_{t}$ is the trap density, and $n_{i}$ is the intrinsic carrier concentration.

$$
I_{\mathrm{dg}}=A_{\mathrm{pn}} W q \sigma v_{\mathrm{th}} N_{t} n_{i} .
$$

The intrinsic carrier concentration can be expanded in (4) where $N_{c}$ is the effective density of state in the conduction band, $N_{v}$ is the effective density of state in the valence band and $E_{g}(T)$ is the energy bandgap, $k$ is Boltzmann's constant, and $T$ is the temperature. The energy bandgap, $E_{g}(T)$, shows a temperature dependence where the bandgap value decreases with increasing temperature [21].

$$
I_{\mathrm{dg}}=A_{\mathrm{pn}} W q \sigma v_{\mathrm{th}} N_{t}\left(N_{c} N_{v}\right)^{1 / 2} \exp \left(-\frac{E_{g}(T)}{2 k T}\right) .
$$

Based on (4), we attempted to extract the energy bandgap from the plot of dark current versus temperature. Taking the natural logarithm of (4), the temperature dependence of the dark current can be expressed in (5). The slope of the $I_{\mathrm{d}}$ versus $1 / k T$ plot shown in Figure 7 was equal to $-E_{g}(T) / 2$. The energy bandgap was estimated to be about $0.71 \mathrm{eV}$, close to that for the InGaAs. It was noted that the bandgap energy of $\mathrm{In}_{0.53} \mathrm{Ga}_{0.47}$ As was reported to be $0.75 \mathrm{eV}$ at $295 \mathrm{~K}[22,23]$. The slight difference between our value and others was likely attributable to the variance from junction temperature and test measurement.

$$
\ln \left(I_{\mathrm{d}}\right) \sim\left(-\frac{E_{g}(T)}{2}\right)\left(\frac{1}{k T}\right) .
$$

\section{Conclusion}

We have extensively studied the temperature dependence of mesa-type APD. In the avalanche breakdown regime 
$\left(|V|>\left|V_{\text {br }}\right|\right)$, our APD device showed excellent temperature stability in breakdown. The breakdown voltage showed very small increase with increasing temperature. The temperature coefficient of the breakdown voltage was found to be $0.017 \mathrm{~V} /{ }^{\circ} \mathrm{C}$, corresponding to a normalized temperature coefficient of $5.1 \times 10^{-4 \circ} \mathrm{C}^{-1}$. We attributed the superior temperature dependence of the avalanche breakdown voltage to the optimized design of the InAlAs multiplication layer thickness and InAlAs charge control layer doping. For the voltage below the breakdown $\left(|V|<\left|V_{\text {br }}\right|\right)$, the dark current increased exponentially with increasing temperature. From the plot of $\ln \left(I_{\mathrm{d}}\right)$ versus $1 / k T$, the energy bandgap extracted from the slope was estimated to be about $0.71 \mathrm{eV}$, in good agreement with the bandgap of InGaAs $(\sim 0.75 \mathrm{eV})$.

\section{Competing Interests}

The authors declare that there is no conflict of interests regarding the publication of this paper.

\section{Acknowledgments}

The authors would like to thank Professor J.-W. Shi from National Central University (Zhongli, Taiwan) for helpful discussions.

\section{References}

[1] E. Ishimura, E. Yagyu, M. Nakaji et al., "Degradation mode analysis on highly reliable guardring-free planar InAlAs avalanche photodiodes," Journal of Lightwave Technology, vol. 25, no. 12, pp. 3686-3693, 2007.

[2] M. Achouche, G. Glastre, C. Caillaud, M. Lahrichi, M. Chtioui, and D. Carpentier, "InGaAs communication photodiodes: from low- to high-power-level designs," IEEE Photonics Journal, vol. 2, no. 3, pp. 460-468, 2010.

[3] H. S. Kim, J. H. Choi, H. M. Bang et al., "Dark current reduction in APD with BCB passivation," Electronics Letters, vol. 37, no. 7, pp. 455-457, 2001.

[4] T. Takeshita, Y. Hirota, T. Ishibashi et al., "Degradation behavior of avalanche photodiodes with a mesa structure observed using a digital OBIC monitor," IEEE Transactions on Electron Devices, vol. 53, no. 7, pp. 1567-1574, 2006.

[5] I. Watanabe, M. Tsuji, M. Hayashi, K. Makita, and K. Taguchi, "Reliability of mesa-structure InAlGaAs-InAlAs superlattice avalanche photodiodes," IEEE Photonics Technology Letters, vol. 8, no. 6, pp. 824-826, 1996.

[6] G. M. Smith, K. A. McIntosh, J. P. Donnelly, J. E. Funk, L. J. Mahoney, and S. Verghese, "Reliable InP-based Geiger-mode avalanche photodiode arrays," in 3th Advanced Photon Counting Techniques, vol. 7320 of Proceedings of SPIE, pp. 1-10, Orlando, Fla, USA, April 2009.

[7] T. P. Pearsall, "Impact ionization rates for electrons and holes in $\mathrm{Ga}_{0.47} \mathrm{In}_{0.53}$ As," Applied Physics Letters, vol. 36, no. 3, pp. 218220, 1980 .

[8] F. Osaka and T. Mikawa, "Excess noise design of InP/ GaInAsP/GaInAs avalanche photodiodes," IEEE Journal of Quantum Electronics, vol. 22, no. 3, pp. 471-478, 1986.

[9] C. L. F. Ma, M. J. Deen, and L. E. Tarof, "A fast and accurate method of extracting two critical device parameters of SAGCM
InP/InGaAs avalanche photodiodes," in Proceedings of the 24th European Solid State Device Research Conference (ESSDERC '94), pp. 459-462, IEEE, September 1994.

[10] J. S. Huang, Y. Jan, H. S. Chen, H. S. Chang, C. J. Ni, and E. Chou, "Predictive reliability model of 10G/25G mesa-type avalanche photodiode degradation," Applied Physics Research, vol. 8, no. 3, pp. 66-74, 2016.

[11] C. L. F. Ma, M. J. Deen, L. E. Tarof, and J. Yu, "Modelling of breakdown voltage and its temperature dependence in SAGCM InP/InGaAs avalanche photodiodes," in Proceedings of the IEEE Electron Devices Meeting (IEDM '94), pp. 22.5.1-22.5.4, San Francisco, Calif, USA, December 1994.

[12] C. R. Viswanathan, "Physical principles of semiconductor devices," UCLA, EE class note, pp. 172-208, 1993.

[13] C. Hu, PN and Metal-Semiconductor Junctions, chapter 4, EE Class Note, Berkeley, Calif, USA, 2009.

[14] T. Bendib, L. Pancheri, F. Dieffal, and G.-F. D. Betta, "Impact of temperature and doping concentration on avalanche photodiode characteristics," in Proceedings of the World Congress on Engineering, vol. 1, pp. 5-8, WCE, London, UK, July 2014.

[15] D. J. Massey, J. P. R. David, and G. J. Rees, “Temperature dependence of impact ionization in submicrometer silicon devices," IEEE Transactions on Electron Devices, vol. 53, no. 9, pp. 23282334, 2006.

[16] M. Singh Tyagi, "Zener and avalanche breakdown in silicon alloyed p-n junctions-I. Analysis of reverse characteristics," Solid State Electronics, vol. 11, no. 1, pp. 99-115, 1968.

[17] L. J. J. Tan, D. S. G. Ong, J. S. Ng et al., "Temperature dependence of avalanche breakdown in InP and InAlAs," IEEE Journal of Quantum Electronics, vol. 46, no. 8, pp. 1153-1157, 2010.

[18] K. Ohnaka, M. Kubo, and J. Shibata, "A low dark current InGaAs/InP p-i-n photodiode with covered mesa structure," IEEE Transactions on Electron Devices, vol. 34, no. 2, pp. 199204, 1987.

[19] M. Fukuda, "Optical semiconductor devices," in Photodiodes, chapter 4, John Wiley \& Sons, New York, NY, USA, 1999.

[20] S. R. Forrest, R. F. Leheny, R. E. Nahory, and M. A. Pollack, " $\mathrm{In}_{0.53} \mathrm{Ga}_{0.47}$ As photodiodes with dark current limited by generation-recombination and tunneling," Applied Physics Letters, vol. 37, no. 3, pp. 322-325, 1980.

[21] J. D. Thomson, H. D. Summers, P. M. Smowton, E. Herrmann, P. Blood, and M. Hopkinson, "Temperature dependence of the lasing wavelength of InGaAs quantum dot lasers," Journal of Applied Physics, vol. 90, no. 9, pp. 4859-4861, 2001.

[22] T. P. Pearsall, “ $G a_{047} \ln _{053}$ As: a ternary semiconductor for photodetector applications," IEEE Journal of Quantum Electronics, vol. 16, no. 7, pp. 709-720, 1980.

[23] T. P. Pearsall, L. Eaves, and J. C. Portal, "Photoluminescence and impurity concentration in $\mathrm{Ga}_{x} \mathrm{In}_{1-x} \mathrm{As}_{y} \mathrm{P}_{1-y}$ alloys latticematched to InP," Journal of Applied Physics, vol. 54, no. 2, pp. 1037-1047, 1983. 


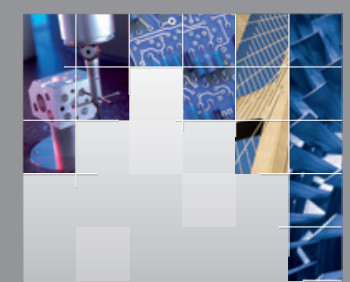

\section{Enfincering}
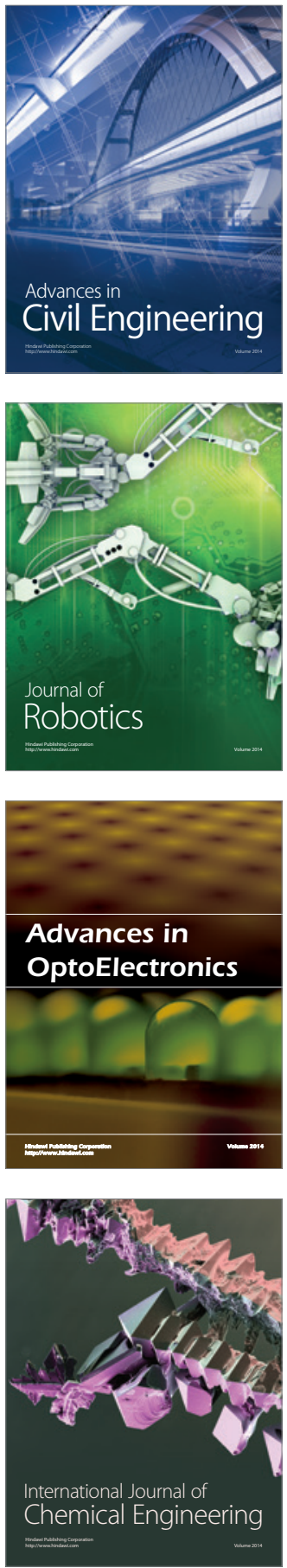

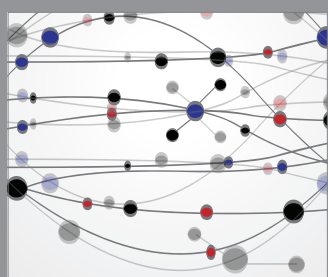

The Scientific World Journal

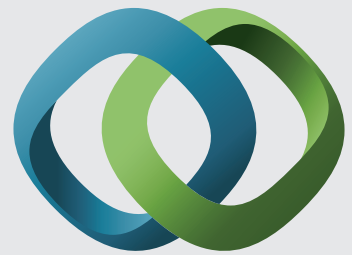

\section{Hindawi}

Submit your manuscripts at

https://www.hindawi.com
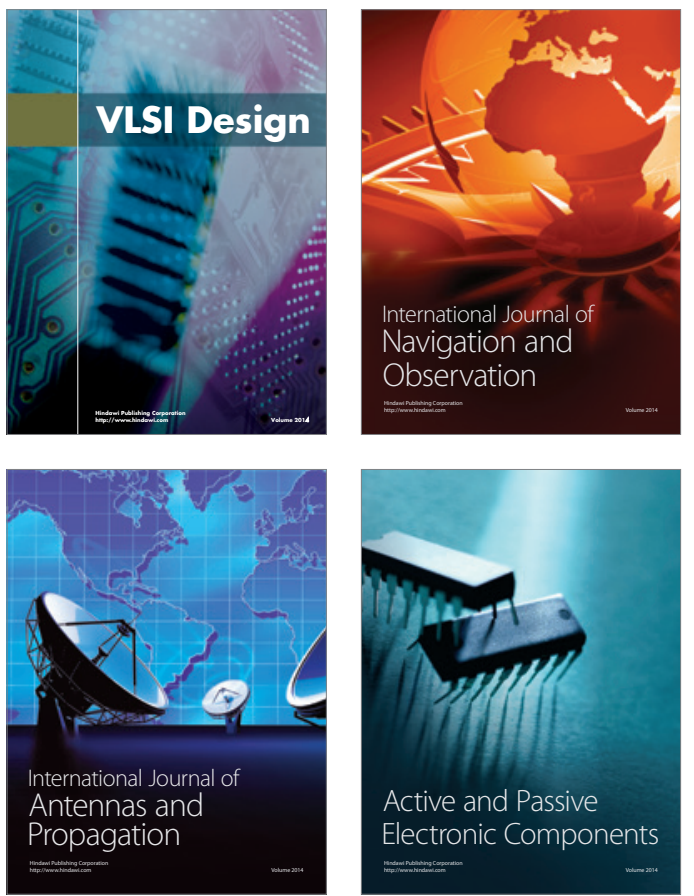
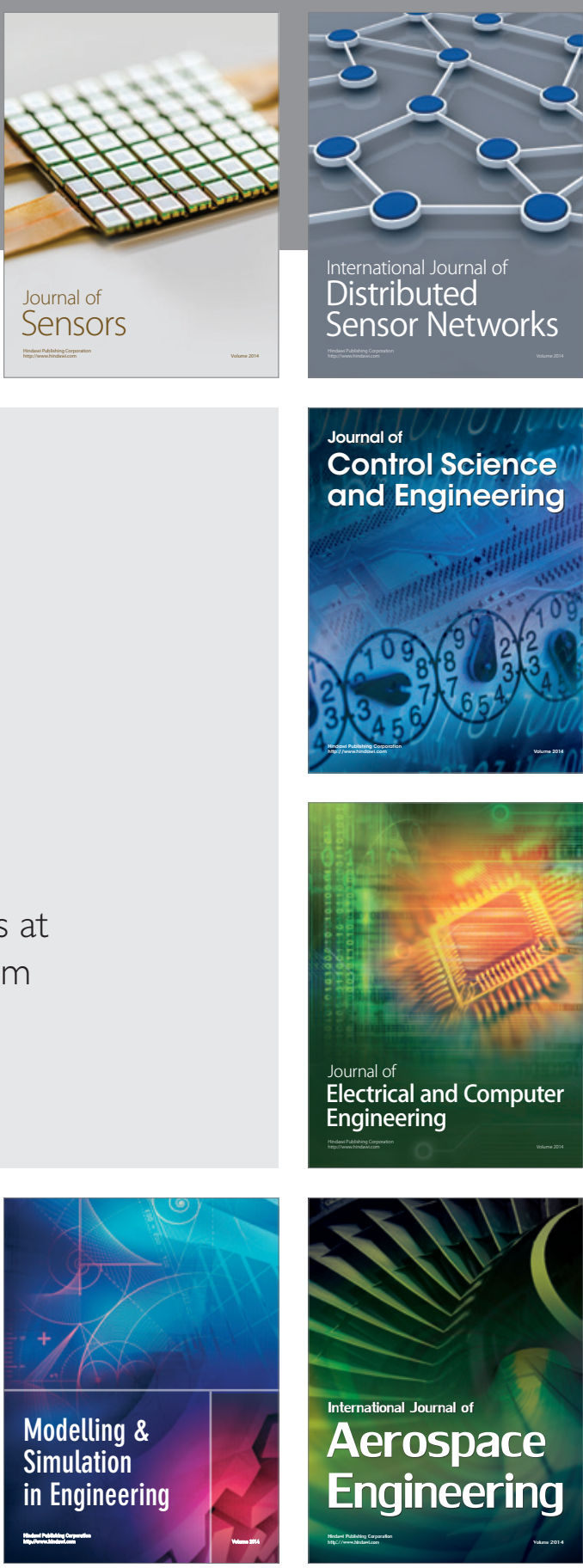

International Journal of

Distributed

Sensor Networks

$-$

Joumal of

Control Science

and Engineering
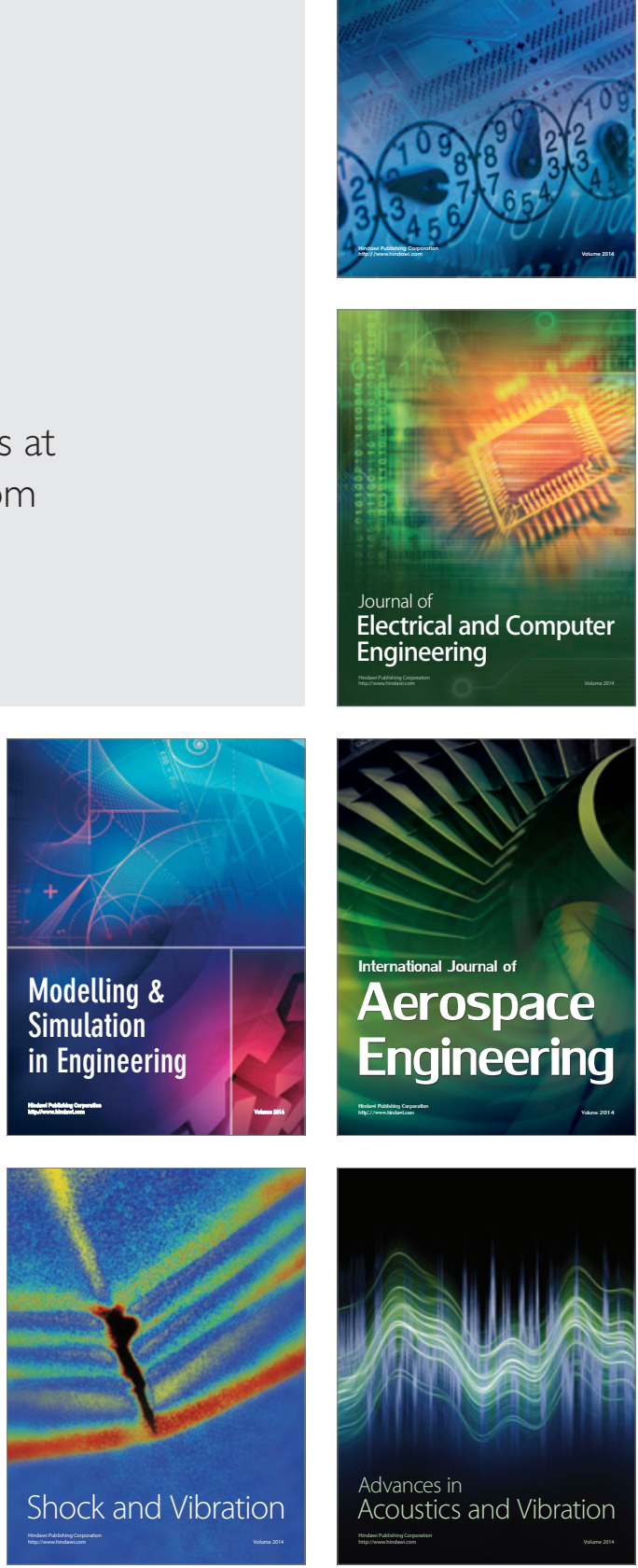